\title{
CDR2 antigen and Yo antibodies
}

\author{
Cecilie Totland $\cdot$ Nina K. Aarskog $\cdot$ Tilo W. Eichler $\cdot$ Mette Haugen • \\ Jane K. Nøstbakken • Sissel E. Monstad • Helga B. Salvesen - Sverre Mørk • \\ Bjørn I. Haukanes • Christian A. Vedeler
}

Received: 3 June 2010/ Accepted: 30 October 2010/Published online: 16 November 2010

(C) The Author(s) 2010. This article is published with open access at Springerlink.com

\begin{abstract}
Paraneoplastic cerebellar degeneration (PCD) is often associated with Yo antibodies that are directed against human cerebellar degeneration-related protein 2 (CDR2). Such antibodies may also be found in ovarian cancer patients without PCD. We studied if there was an association between Yo antibody production and differences in CDR2 cDNA sequence, mRNA or CDR2 expression in ovarian cancers. We found similar CDR2 cDNA sequence, mRNA and protein levels in primary ovarian cancers, with or without associated Yo antibodies. CDR2 was also present in other cancers, as well as in normal ovary tissue. The results suggest that Yo antibodies are not only related to the expression of CDR2 alone, but also to immune dysregulation.
\end{abstract}

C. Totland · N. K. Aarskog - T. W. Eichler - M. Haugen

J. K. Nøstbakken · S. E. Monstad · C. A. Vedeler ( $\square)$

Department of Neurology, Haukeland University Hospital,

Bergen, Norway

e-mail: christian.vedeler@helse-bergen.no

H. B. Salvesen

Department of Gynaecology, Haukeland University Hospital, Bergen, Norway

S. Mørk

Department of Pathology, Haukeland University Hospital, Bergen, Norway

\section{B. I. Haukanes}

Center for Medical Genetics and Molecular Medicine,

Haukeland University Hospital, Bergen, Norway

H. B. Salvesen - C. A. Vedeler

Department of Clinical Medicine, Haukeland University

Hospital, Bergen, Norway

S. Mørk

Gades Institute, University of Bergen, Bergen, Norway
Keywords Ovarian cancer - CDR2 - Yo antibody · Paraneoplastic cerebellar degeneration

\section{Introduction}

Paraneoplastic cerebellar degeneration (PCD) is often related to breast and ovarian tumors, which aberrantly express an onconeural antigen called cerebellar degeneration-related protein 2 (CDR2) [1,2]. Although CDR2 is a widely expressed gene at the mRNA level, post-transcriptional mechanisms have been reported to confine the expression of CDR2 protein to mouse cerebellum and testis [3]. CDR2 protein has been shown to be present in $62 \%$ of ovarian cancers, but not in normal ovary tissue [4].

The biological function of CDR2 is partly characterized, as CDR2 through its leucine zipper motif has been demonstrated to interact with c-myc [5], with cell cycle-related proteins $[6,7]$ and with a protein kinase [8], indicating that CDR2 is involved in signal transduction and gene transcription. Furthermore, CDR2 has been found to attenuate hypoxic response in renal cell carcinoma [9], and recent data suggest a role for CDR2 and c-myc in mitosis in cycling cells [10].

Patients with PCD often harbor Yo antibodies and CDR2-specific cytotoxic lymphocytes in the blood and cerebrospinal fluid. It is, therefore, proposed that both humoral and cellular immune mechanisms participate in the pathogenesis of PCD, leading to necrosis or apoptosis of Purkinje cells $[1,2,11]$. The role of Yo antibodies in PCD is uncertain, as such antibodies are also observed in cancer patients without paraneoplastic neurological syndromes [12, 13]. Recently, Yo antibodies have been linked to CCDC104 antibodies, but the function of CCDC104 is largely unknown [14]. 
How Yo antibody synthesis is regulated in some patients with cancer is unknown. We have, therefore, studied whether the production of Yo antibodies could be related to variants in the CDR2-coding sequence or to differences in the mRNA or protein levels of CDR2 in ovarian cancer. Furthermore, as previous results have suggested that CDR2 is only present in ovarian cancers and not in normal ovary tissue [4], we also studied whether there could be posttranscriptional differences in such tissue.

\section{Materials and methods}

Tissue and serum samples

Ovarian cancers were obtained from 16 patients aged 41-79 years operated at the Department of Gynaecology, Haukeland University Hospital (Table 1). None of the patients received chemotherapy or immunotherapy before the operation. Two of the patients had PCD. From these two patients only formalin-fixed cancer tissue was available. Colon cancer was obtained from a patient without paraneoplastic neurological syndrome. Normal human cerebrum and cerebellum were obtained from autopsy of a patient without neurological disorder. The following cancer cell lines were obtained from ATCC (www.lgcstandardsatcc.org): lung cancer (A549), ovarian cancer (SK-OV-3), neuroblastoma (SK-N-SH), and cervical cancer (HeLa). Tissue and cell lysates were made using total protein extraction kit from Chemicon (www.chemicon.com). Lysates from human ovary tumor and human normal ovary tissue were purchased from Prosci Inc (www.prosciinc.com). Recombinant CDR2 protein was produced in a coupled transcription/translation (ITT) system with a T7 RNA polymerase and nuclease-treated rabbit reticulocyte lysate, and CDR2-negative lysate was prepared with the ITT reaction without plasmid [12, 13]. Protein concentrations were determined using a BioRad DC protein assay (www.biorad.com). Tissue and lysates were kept at $-80^{\circ} \mathrm{C}$ and serum samples at $-20^{\circ} \mathrm{C}$. Yo antibodies have previously been found in 3 of 182 sera from patients with ovarian cancer at the Department of Gynaecology, Haukeland University Hospital, and the 14 patients included in this report were part of this study [13]. In addition, we also included two patients with PCD and Yo antibodies.

RNA purification and cDNA synthesis

Total RNA was extracted from 14 ovarian cancers by homogenization in a Trizol reagent (www.invitrogen.com) and RTL buffer containing $\beta$-mercaptoethanol followed by RNeasy mini-kit (www.qiagen.com). We also used a panel of human total RNA from 11 different types of tissue (brain, heart, kidney, liver, lung, trachea, mammary gland, prostate, skeletal muscle, testis and uterus), as well as human reference total RNA (www.clontech.com). RNA was stored at $-80^{\circ} \mathrm{C}$ until use. From each of the samples, we reverse transcribed $100 \mathrm{ng}$ of RNA to cDNA using TaqMan RT reagents according to the manufacturer's instructions (www.appliedbiosystems.com).
Table 1 Patients with ovarian cancer and presence of $\mathrm{Yo}_{\mathrm{O}}$ antibodies and CDR2 mRNA and protein

\footnotetext{
${ }^{a}$ Patients with paraneoplastic cerebellar degeneration; $C D R 2$ cerebellar degeneration-related protein 2; FIGO International Federation of Gynecology and Obstetrics
}

\begin{tabular}{lllllll}
\hline $\begin{array}{l}\text { Patient } \\
\text { no. }\end{array}$ & $\begin{array}{l}\text { Age } \\
\text { (years) }\end{array}$ & $\begin{array}{l}\text { Histology } \\
\text { (adenocarcinoma) }\end{array}$ & $\begin{array}{l}\text { FIGO } \\
\text { stage }\end{array}$ & $\begin{array}{l}\text { Yo } \\
\text { antibody }\end{array}$ & $\begin{array}{l}\text { CDR2 } \\
\text { mRNA }\end{array}$ & $\begin{array}{l}\text { CDR2 } \\
\text { protein }\end{array}$ \\
\hline 1 & 78 & Endometrioid & III & + & + & + \\
2 & 55 & Serous & III & - & + & + \\
3 & 57 & Serous & III & - & + & + \\
4 & 53 & Mucinous/borderline & III & - & + & + \\
5 & 64 & Serous & III & - & + & + \\
6 & 69 & Undifferentiated & III & - & + & + \\
7 & 58 & Serous & IV & + & + & + \\
8 & 78 & Serous & IV & - & + & + \\
9 & 56 & Serous & III & - & + & + \\
10 & 48 & Endometrioid & III & - & + & + \\
11 & 62 & Endometrioid & III & + & + & + \\
12 & 79 & Mucinous & I & - & + & + \\
13 & 41 & Mucinous/borderline & I & - & + & + \\
14 & 55 & Clear cell & I & - & + & + \\
$15^{\text {a }}$ & 60 & Serous & III & + & ND & + \\
$16^{\text {a }}$ & 58 & Serous & IV & + & ND & + \\
\hline
\end{tabular}


Real-time quantitative polymerase chain reaction

Real-time quantitative polymerase chain reaction (RTPCR) was used to quantify mRNA expression in the 14 ovarian cancers, the panel of human total RNA and the human reference total RNA. All primers and probes were obtained from Assay-on-Demand gene expression products at Applied Biosystems (www.appliedbiosystems.com). As endogenous control genes, we used human large ribosomal protein (RPLPO), human transcription factor IID/TATA box-binding protein (TBP) and the housekeeping gene human glyceraldehyde phosphate dehydrogenase (GAP$\mathrm{DH}$ ), including the test gene CDR2. We performed the RTPCR in the ABI prism 7900 Sequence Detection System run by standard PCR cycling conditions. The relative quantification of mRNA expression was based on the standard curve method (User Bulletin \#2, Applied Biosystems). Human reference total RNA (www.clontech.com) was used to generate the standard curve. We included a "no template control" in each run. Each sample was run and analyzed in triplicate.

The expression of CDR2 was normalized to all the three reference genes (PO, TBP, and GAPDH). The data were transferred to Graphpad Prism, version 3.0 (www.graph pad.com) and calculated using Mann-Whitney nonparametric $U$-test. $P<0.05$ was considered statistically significant. We assessed the three reference genes to evaluate the validity of the normalization of the CDR2 expression. All the three reference genes were highly significantly correlated $(r=0.50-0.65, P<0.008-0.0002)$ according to the Spearman rank correlation test (Graphpad Prism).

\section{Sequencing}

CDR2 cDNA was sequenced from the 14 ovarian cancers. The following primers for amplifying CDR2 were used: 293U20 5'-CTA GAA GAC CCA GCC GAG AT-3', 659L19 5'-TTG CAG GCA TTC AAT CGT T-3', 293u21 5'-CTA GAA GAC CCA GCC GAG ATG-3', 975L21 5'-CCT TTA ACA CGA GCC CAT ATT-3', 874U20 5'-GCC CTG ATG AAG AGG AAA AT-3', 1370L20 5'-GCT GTA CTG CGT GTC CAC TT-3', $1265 \mathrm{U} 29$ 5'-AGG GAG TGA CAT CGT GAA GG-3', and 1819L20 5'-CCT CGT TGT ATG CAT TTG CT-3'. We used one tenth cDNA as a template for a $\mathrm{PCR}$ reaction using Amplitaq Gold polymerase (www.appliedbiosystems.com). cDNA was denaturated at $95^{\circ} \mathrm{C}$ for $10 \mathrm{~min}$ followed by 35 PCR cycles $\left(95^{\circ} \mathrm{C}\right.$ for $30 \mathrm{~s}, 55^{\circ} \mathrm{C}$ for $30 \mathrm{~s}$, and $72^{\circ} \mathrm{C}$ for $60 \mathrm{~s}$ ) and then held at $4^{\circ} \mathrm{C}$. The PCR product was purified using Montage PCR Centrifugal Filter Devices according to the manufacturer's instructions (www.Millipore.com). The PCR product was bidirectionally sequenced using a
Big Dye terminator 3.1 cycle sequencing kit according to the manufacturer's recommendation on an ABI 3700 DNA Analyzer (www.appliedbiosystems.com). ChromasPro was used to analyze the sequence files (www.softpedia.com), BLAST for identifying the DNA sequence (www.ncbi.nml. nih.gov), and Clustal W2 for aligning sequences (www.ebi.ac.uk/Tools/clustalw2).

\section{Immunoblot}

We subjected the various extracts to a $10 \%$ SDS-PAGE and performed western blotting. The following concentrations were loaded to the gels: $10 \mu \mathrm{g}$ of each cancer extract, $20 \mu \mathrm{g}$ of the ovarian lysates according to the manufacturer (www.prosci-inc.com), and $100 \mu \mathrm{g}$ of the human cerebrum and cerebellum extracts. The membranes were first incubated with a 1:200 dilution of the anti-CDR2 monoclonal antibody (sc-100320) and then with a goat anti-mouse HRP antibody (sc-2005) diluted 1:1000, both antibodies obtained from Santa Cruz Biotechnology, Inc. (www.scbt. com). The reactions were developed by the ECL Plus Western Blotting Detection system (RPN2132) (www. healthcare.com) and then read using a Fuji-las 1000 luminescent image analyzer (www.fujifilm.com).

\section{Immunohistochemistry}

Intracellular localization of CDR2 was done in the ovarian, lung, and cervical cancer cell lines. The cell lines were grown and treated as previously described [10]. After fixation and permeabilization, the cells were incubated with a 1:200 dilution of anti-CDR2 polyclonal antibody (ab59566) or anti-CDR2 monoclonal antibody (sc-100320) (www.abcam.com) and then with Alexa Fluor 488 goat anti-rabbit antibody (A-11008) or with Alexa Fluor 488goat anti-mouse antibody (A-11001) (www.probes. invitrogen.com), respectively, diluted 1:200 in blocking solution. The slides were mounted with Vectashield with or without DAPI (www.vectorlabs.com). Indirect immunofluorescence was done using Zeiss LSM 510 Meta. Absorption experiments were performed as previously described [15] by diluting the anti-CDR2 polyclonal antibody to one titer below the highest dilution still producing staining of HeLa cells. The antibody was incubated with equal volume of the recombinant CDR2 protein overnight. The antibody-antigen complex was spun down, and the supernatant was applied to the cells as described and analyzed at a Zeiss Axiovert M200.

Intracellular localization of CDR2 was also done in three ovarian cancer biopsies (from patients 1,15 , and 16 in Table 1) and in one colon cancer biopsy using the antiCDR2 polyclonal antibody (ab59566) (www.abcam.com) diluted 1:400. The immunoperoxidase staining followed 


\section{A}

$62 \mathrm{kDa}$

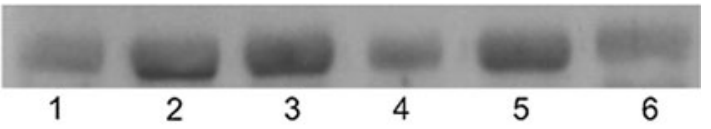

\section{B} $62 \mathrm{kDa}$

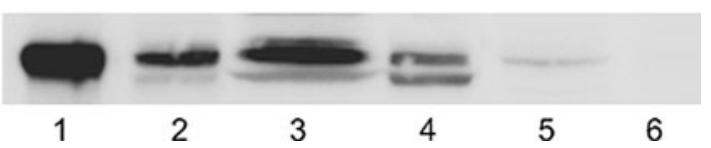

Fig. 1 a Western blot shows CDR2 in four human ovarian cancer extracts (1-4), in lysates of human ovarian cancer (5), and human normal ovary tissue (6). b Western blot shows recombinant CDR2 as positive control (1), CDR2 in cell lines of lung cancer (2), ovarian cancer (3), neuroblastoma (4), and in human normal cerebellum (5), but not in human normal cerebrum $(6)$

standard procedures at the Department of Pathology, Haukeland University Hospital.

\section{Results}

Table 1 presents the histology and International Federation of Gynecology and Obstetrics (FIGO) stage of the ovarian cancers. Five of the 16 ovarian cancer patients had Yo antibodies. The other 11 cancer patients were Yo antibody negative.
Sequencing of CDR2 cDNA revealed no variants in the 14 ovarian cancers. For reference, we used CDR2 cDNA sequence from human CDR2 (62 kDa), NM_001802 (www.ncbi.nlm.nih.gov).

Using RT-PCR, we found approximately similar expression levels (1-2 fold differences) in the mRNA CDR2 between the 14 ovarian cancers and in the other normal human tissue controls-brain, heart, kidney, liver, lung, trachea, mammary gland, prostate, skeletal muscle, testis, and uterus - and in the human reference total RNA.

Immunoblot of the 14 human ovarian cancer extracts, as well as from the human ovary tumor and human normal ovary tissue lysates showed bands of approximately similar intensity with a molecular weight of $62 \mathrm{kDa}$ (Fig. 1a). The reaction in immunoblot was stronger with ovarian cancer cells compared to lung and neuroblastoma cells. A weak band of $62 \mathrm{kDa}$ was found in the cerebellar, but not in the cerebrum extracts (Fig. 1b). We used recombinant CDR2 as positive control. This band also corresponded to $62 \mathrm{kDa}$, whereas no binding of the monoclonal anti-CDR2 antibody was found to the negative ITT lysate. There was no binding of the secondary antibody alone to any of the extracts or lysates.

The polyclonal anti-CDR2 antibody showed granular staining mainly of the cytoplasm of the ovarian, lung, and cervical cancer cell lines (Fig. 2a, b, and d).
Fig. 2 Staining with polyclonal antibody against CDR2 shows granular green staining mainly in the cytoplasm of cell lines of ovarian cancer (a) and lung cancer (b). DAPI stains the nuclei blue. A similar granular staining pattern was found in the cell line of cervical cancer using monoclonal antibody against CDR2 (c) and polyclonal antibody against CDR2 (d), but the staining was almost completely abolished after preincubation of the polyclonal antibody with recombinant CDR2 (e)
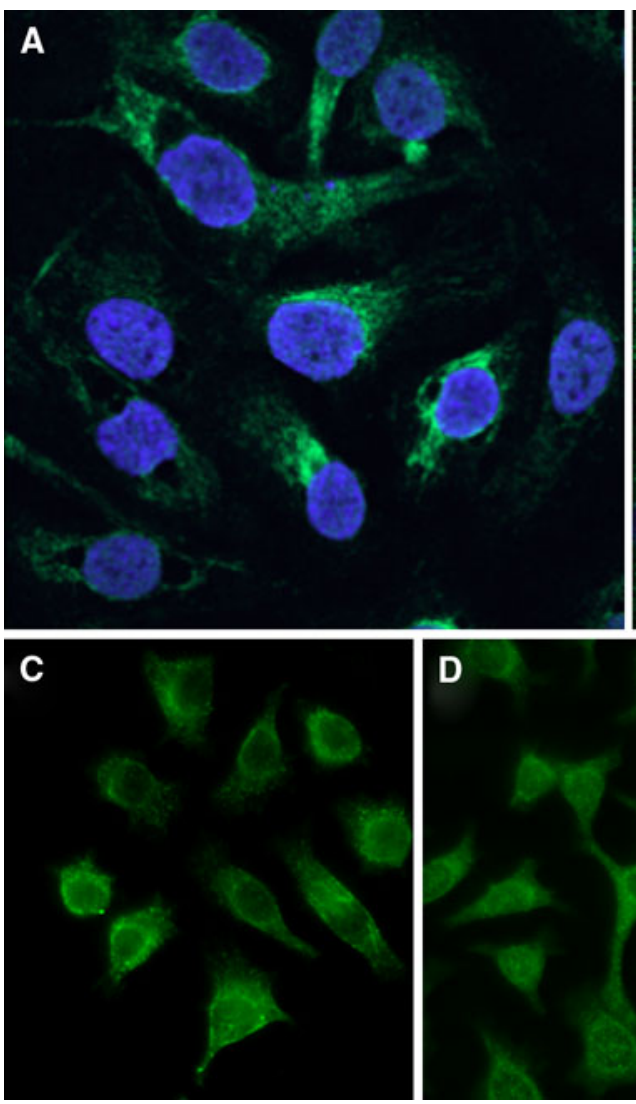
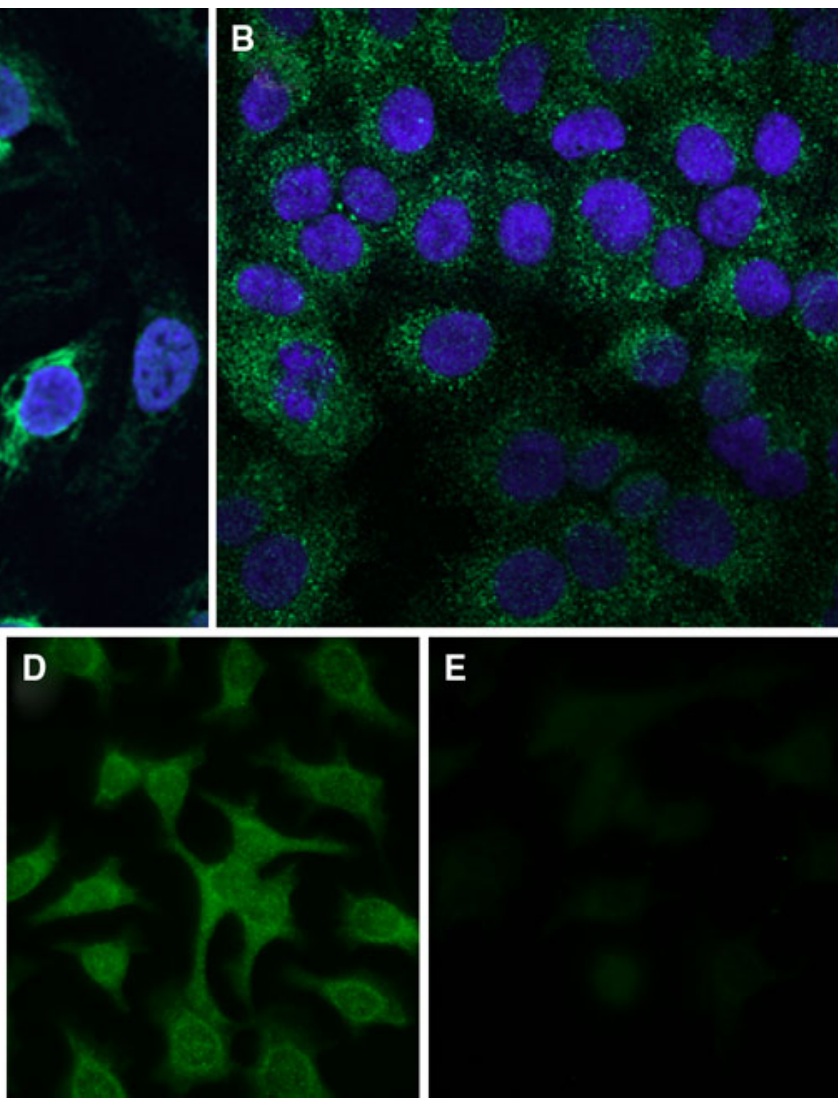

E

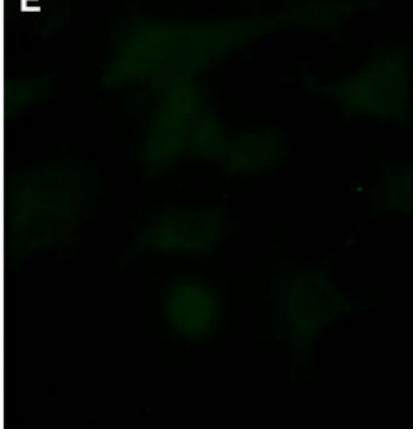


Fig. 3 Ovarian and colon cancer biopsies were stained with polyclonal antibody against CDR2. There was no binding of the secondary antibody alone to ovarian cancer (a). The same ovarian cancer from a patient with $\mathrm{Yo}_{0}$ antibodies, but no PCD showed CDR2 staining of cytoplasm and nuclei of the cancer cells (b). This biopsy also contained normal ovarian tissue with slight CDR2 staining of stromal cells and strong CDR2 staining of epithelial cell (c). Two other ovarian cancers from patients with PCD and Yo antibodies showed nuclear CDR2 staining of the cancer cells $(\mathbf{d}, \mathbf{e})$. Colon cancer cells also expressed nuclear and cytoplasmic CDR2 staining (f)
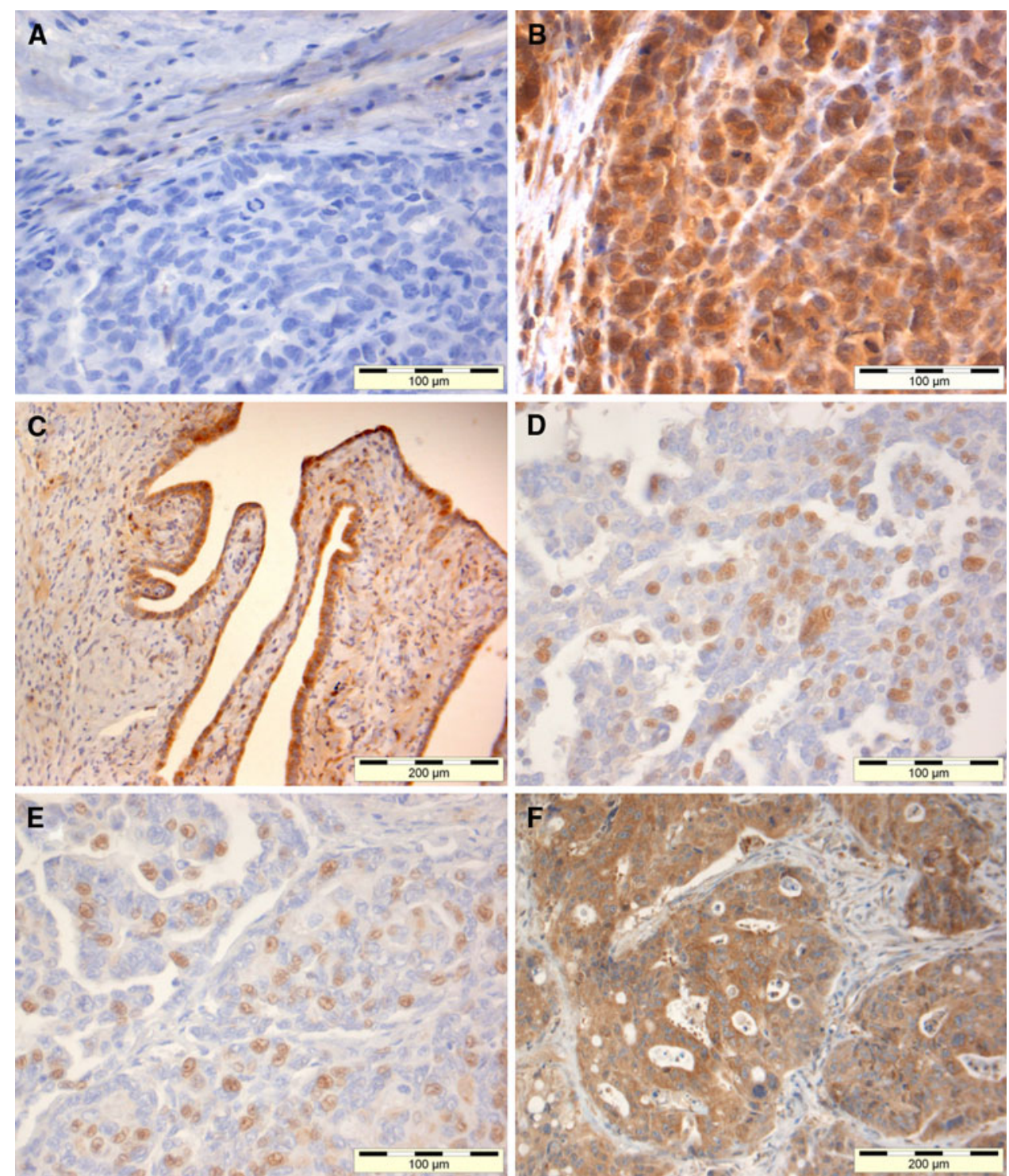

The monoclonal antibody against CDR2 showed a similar staining pattern (Fig. 2c compared to d). Preabsorption of the polyclonal antibody with recombinant CDR2 almost completely abolished the staining (Fig. 2d compared to e). The polyclonal anti-CDR2 antibody showed a nuclear and cytoplasmic staining of cancer cells in ovarian and colon biopsies. Staining was also present on normal stromal and epithelial cells present in ovarian cancer. There was no binding of the secondary antibody alone to any of the tissue sections or cell slides (Fig. 3). The monoclonal anti-CDR2 antibody did not stain the tissue sections.

\section{Discussion}

This study provides further data on CDR2 in various cancers and its relation to Yo antibodies. We found that sequencing CDR2 cDNA from various human ovarian cancers showed no variants that could involve possible alternative transcription of the CDR2 gene. Whether there are any variants in the CDR2 promoter region is not known. However, mutations in another neuro-oncogene $(\mathrm{HuD})$ have been demonstrated in lung neuroendocrine tumors [16], and variable levels of expression of the $\mathrm{HuD}$ gene were previously reported in small-cell lung cancer cell lines with different degrees of neuroendocrine differentiation [17]. It may, therefore, be differences in the gene regulation between CDR2 and HuD.

The human ovarian cancers and various normal human tissues expressed approximately similar levels of CDR2 mRNA. This is in accordance with a previous study of mouse tissue in which CDR2 mRNA was widely expressed using Northern blot analysis [3]. 
We also found that the ovarian cancers expressed approximately similar levels of CDR2 protein regardless of histology or FIGO stage. However, the material has to be larger to be certain if there are significant differences in CDR2 mRNA or protein expression between histological subtypes of ovarian cancer. Recent findings have suggested that human papillary renal cell carcinoma expresses higher CDR2 mRNA and protein levels than human clear-cell renal carcinoma [9]. In our study, we also detected CDR2 protein in cell lines of lung, cervical and ovarian cancer and neuroblastoma, as well as in colon cancer, indicating that CDR2 protein is expressed in various cancers.

In a previous study, only 13 of $21(62 \%)$ human ovarian cancers (20 epithelial cancers and one stromal cancer) expressed Yo protein using immunoblot of the cancer lysates and serum from a PCD patient containing Yo antibodies [4]. Our results, however, showed that CDR2 was present in all 16 ovarian cancers studied. The specificity and sensitivity of the patient Yo serum used in the previous study [4] and the monoclonal and polyclonal antiCDR2 antibodies used in this study are probably different, which could explain the difference in the results. Based on our results, we suggest that CDR2 translation occurs in all ovarian cancers, but larger studies are needed to confirm this. Our results are in accordance with findings in smallcell lung cancer, where all cancers expressed the paraneoplastic antigen HuD [18]. This was also true for CDR2 in papillary renal cell carcinoma, but in clear-cell renal carcinoma about $1 \%$ was not stained for CDR2 [9].

We found by immunoblot that CDR2 was expressed with higher intensity in ovarian, than in lung cancer cells, but immunofluorescence studies of ovarian, cervical, and lung cancer cell lines revealed approximately similar CDR2 granular staining which was mainly localized to the cytoplasm. A similar staining pattern has recently also been demonstrated in cervical cancer cells, and the results further showed that CDR2 was colocalized with c-myc during mitosis [10]. However, in tissue sections of ovarian and colon cancers we found both nuclear and cytoplasmic CDR2 staining. The CDR2 staining was also present in ovarian cancers from patients with or without PCD.

We found that CDR2 was not only present in various cancers, but also in normal ovary tissue. This is in accordance with results from the Human Protein Atlas where CDR2 has been reported in normal ovary follicle and stromal cells (www.proteinatlas.org). However, it contrasts previous observations where Yo protein was not detected by immunoblot of normal ovary lysate using serum from a PCD patient [4], but the explanation for the different results may again be the specificity and sensitivity of the antibodies used.

We have previously reported that Yo antibodies are not related to ovarian cancer histology or FIGO stage [12]. The present results indicate that there is no direct association between CDR2 cDNA sequence, mRNA, or protein level and Yo antibodies. Unfortunately, we did not have more Yo positive sera available from patients with corresponding ovary cancers to be studied as outlined above. However, we also found CDR2 in ovary cancers from patients with and without PCD, in various other cancers, as well as in normal ovary tissue.

Our findings, therefore, suggest that Yo antibody synthesis and development of PCD is not only related to the expression of CDR2 alone, but also to immune dysregulations, such as antigen presentation and $\mathrm{T}$ and $\mathrm{B}$ cell cooperation. This is supported by recent findings that specific disease-associated HLA class II alleles [19], as well as dysfunction of regulatory T cells [20] may play a role in the pathogenesis of paraneoplastic neurological syndromes. Taken together, the results further strengthen the hypothesis that paraneoplastic neurological syndromes are autoimmune diseases.

Acknowledgments We thank the Western Norway Regional Health Authority (Helse Vest), Gerda Meyer Nyquist Guldbranson and Gerdt Meyer Nyquist legacy and the Norwegian Cancer Society (Harald Andersens legat) for financial support and Britt Edvardsen for technical assistance. The confocal imaging was performed at the Molecular Imaging Center (Fuge, Norwegian Research Council), University of Bergen.

Conflict of interest The authors declare that they have no conflicts of interest.

Open Access This article is distributed under the terms of the Creative Commons Attribution Noncommercial License which permits any noncommercial use, distribution, and reproduction in any medium, provided the original author(s) and source are credited.

\section{References}

1. Darnell RB, Posner JB (2003) Paraneoplastic syndromes involving the nervous system. N Engl J Med 349:1543-1554

2. Storstein A, Vedeler CA (2007) Paraneoplastic neurological syndromes and onconeural antibodies: clinical and immunological aspects. Adv Clin Chem 44:143-185

3. Corradi JP, Yang C, Darnell JC, Dalmau J, Darnell RB (1997) A post-transcriptional regulatory mechanism restricts expression of the paraneoplastic cerebellar degeneration antigen cdr2 to immune privileged tissues. J Neurosci 17:1406-1415

4. Darnell JC, Albert ML, Darnell RB (2000) Cdr2, a target antigen of naturally occuring human tumor immunity, is widely expressed in gynecological tumors. Cancer Res 60:2136-2139

5. Okano HJ, Park WY, Corradi JP, Darnell RB (1999) The cytoplasmic Purkinje onconeural antigen cdr2 down-regulates c-Myc function: implications for neuronal and tumor cell survival. Genes Dev 13:2087-2097

6. Sakai K, Shirakawa T, Li Y, Kitagawa Y, Hirose G (2002) Interaction of a paraneoplastic cerebellar degeneration-associated neuronal protein with the nuclear helix-loop-helix leucine zipper protein MRG X. Mol Cell Neurosci 19:477-484 
7. Sakai K, Kitagawa Y, Saiki S, Saiki M, Hirose G (2004) Effect of a paraneoplastic cerebellar degeneration-associated neural protein on B-myb promoter activity. Neurobiol Dis 15:529-533

8. Takanaga H, Mukai H, Shibata H, Toshimori M, Ono Y (1998) PKN interacts with a paraneoplastic cerebellar degenerationassociated antigen, which is a potential transcription factor. Exp Cell Res 241:363-372

9. Balamurugan K, Luu VD, Kaufmann MR, Hofmann VS, Boysen G, Barth S, Bordoli MR, Stiehl DP, Moch H, Schraml P, Wenger RH, Camenisch G (2009) Onconeuronal cerebellar degenerationrelated antigen, $\mathrm{Cdr} 2$, is strongly expressed in papillary renal cell carcinoma and leads to attenuated hypoxic response. Oncogene 28:3274-3285

10. O'Donvan KJ, Diedler J, Couture GC, Fak JJ, Darnell RB (2010) The onconeural antigen cdr2 is a novel APC/C target that acts in mitosis to regulate c-myc target genes in mammalian tumor cells. PLoS ONE 5:e10045

11. Storstein A, Krossnes BK, Vedeler CA (2009) Morphological and immunohistochemical characterization of paraneoplastic cerebellar degeneration associated with Yo antibodies. Acta Neurol Scand 120:64-67

12. Monstad SE, Storstein A, Dorum A, Knudsen A, Lonning PE, Salvesen HB, Aarseth JH, Vedeler CA (2006) Yo antibodies in ovarian and breast cancer patients detected by a sensitive immunoprecipitation technique. Clin Exp Immunol 144:53-58

13. Monstad SE, Knudsen A, Salvesen HB, Aarseth JH, Vedeler CA (2009) Onconeural antibodies in sera from patients with various types of tumours. Cancer Immunol Immunother 58:1795-1800
14. Totland C, Bredholt G, Haugen M, Haukanes BI, Vedeler CA (2010) Antibody to CCDC104 is associated with a paraneoplastic antibody to CDR2 (anti-Yo). Cancer Immunol Immunother 59:231-237

15. Storstein A, Knudsen A, Vedeler CA (2005) Proteasome antibodies in paraneoplastic cerebellar degeneration. J Neuroimmunol 165:172-178

16. D'Alessandro V, Muscarella LA, la Torre A, Bisceglia M, Parrella P, Scaramuzzi G, Storlazzi CT, Trombetta D, Kok K, De Cata A, Sperandeo M, Zelante L, Carella M, Vendemiale G (2010) Molecular analysis of the HuD gene in neuroendocrine lung cancers. Lung Cancer 67:69-75

17. King PH (1997) Differential expression of the neuroendocrine genes Hel-N1 and $\mathrm{HuD}$ in small-cell lung carcinoma: evidence for down-regulation of $\mathrm{HuD}$ in the variant phenotype. Int $\mathrm{J}$ Cancer 74:378-382

18. Dalmau J, Graus F, Cheung NK, Rosenblum MK, Ho A, Canete A, Delattre JY, Thompson SJ, Posner JB (1995) Major histocompatibility proteins, anti-Hu antibodies, and paraneoplastic encephalomyelitis in neuroblastoma and small cell lung cancer. Cancer 75:99-109

19. de Graaf MT, de Beukelaar JWK, Haasnoot GW, Levering WHBM, Rogemond V, Didelot A, Honnorat J, Gratama JW, Sillewis Smitt PAE (2010) HLA-DQ2 ${ }^{+}$individuals are susceptible to $\mathrm{Hu}-\mathrm{Ab}$ associated paraneoplastic neurlogical syndromes. J Neuroimmunol 226:147-149

20. Tani T, Tanaka K, Idezuka J, Nishizawa M (2008) Regulatory T cells in paraneoplastic neurological syndromes. J Neuroimmunol 196:166-169 